\title{
\begin{tabular}{l|l} 
Mitraries & DSpace@MIT
\end{tabular}
}

MIT Open Access Articles

Scaling properties at freeze-out in relativistic heavy-ion collisions

The MIT Faculty has made this article openly available. Please share how this access benefits you. Your story matters.

Citation: Aggarwal, M. et al. "Scaling properties at freeze-out in relativistic heavy-ion collisions." Physical Review C 83 (2011). (C2011 American Physical Society.

As Published: http://dx.doi.org/10.1103/PhysRevC.83.034910

Publisher: Institute of Electrical and Electronics Engineers

Persistent URL: http://hdl.handle.net/1721.1/66134

Version: Final published version: final published article, as it appeared in a journal, conference proceedings, or other formally published context

Terms of Use: Article is made available in accordance with the publisher's policy and may be subject to US copyright law. Please refer to the publisher's site for terms of use. 


\section{Scaling properties at freeze-out in relativistic heavy-ion collisions}

M. M. Aggarwal,${ }^{30}$ Z. Ahammed, ${ }^{22}$ A. V. Alakhverdyants, ${ }^{18}$ I. Alekseev, ${ }^{16}$ J. Alford,${ }^{19}$ B. D. Anderson, ${ }^{19}$ C. D. Anson, ${ }^{28}$ D. Arkhipkin, ${ }^{3}$ G. S. Averichev, ${ }^{18}$ J. Balewski, ${ }^{23}$ L. S. Barnby, ${ }^{2}$ D. R. Beavis, ${ }^{3}$ R. Bellwied, ${ }^{50}$ M. J. Betancourt, ${ }^{23}$ R. R. Betts, ${ }^{8}$ A. Bhasin, ${ }^{17}$ A. K. Bhati, ${ }^{30}$ H. Bichsel, ${ }^{49}$ J. Bielcik, ${ }^{10}$ J. Bielcikova, ${ }^{11}$ B. Biritz, ${ }^{6}$ L. C. Bland, ${ }^{3}$ W. Borowski, ${ }^{41}$ J. Bouchet, ${ }^{19}$ E. Braidot, ${ }^{27}$ A. V. Brandin, ${ }^{26}$ A. Bridgeman, ${ }^{1}$ E. Bruna, ${ }^{52}$ S. Bueltmann, ${ }^{29}$ I. Bunzarov, ${ }^{18}$ T. P. Burton, ${ }^{3}$ X. Z. Cai, ${ }^{40}$

H. Caines,${ }^{52}$ M. Calderón de la Barca Sánchez,${ }^{5}$ D. Cebra, ${ }^{5}$ R. Cendejas, ${ }^{6}$ M. C. Cervantes, ${ }^{42}$ Z. Chajecki, ${ }^{28}$ P. Chaloupka, ${ }^{11}$ S. Chattopadhyay, ${ }^{47}$ H. F. Chen, ${ }^{38}$ J. H. Chen, ${ }^{40}$ J. Y. Chen,${ }^{51}$ J. Cheng, ${ }^{44}$ M. Cherney, ${ }^{9}$ A. Chikanian,${ }^{52}$ K. E. Choi, ${ }^{34}$ W. Christie, ${ }^{3}$ P. Chung, ${ }^{11}$ M. J. M. Codrington, ${ }^{42}$ R. Corliss, ${ }^{23}$ J. G. Cramer, ${ }^{49}$ H. J. Crawford, ${ }^{4}$ S. Dash, ${ }^{13}$ A. Davila Leyva, ${ }^{43}$ L. C. De Silva ${ }^{50}$ R. R. Debbe,${ }^{3}$ T. G. Dedovich, ${ }^{18}$ A. A. Derevschikov,${ }^{32}$ R. Derradi de Souza,${ }^{7}$ L. Didenko, ${ }^{3}$ P. Djawotho, ${ }^{42}$ S. M. Dogra, ${ }^{17}$ X. Dong, ${ }^{22}$ J. L. Drachenberg, ${ }^{42}$ J. E. Draper, ${ }^{5}$ J. C. Dunlop, ${ }^{3}$ M. R. Dutta Mazumdar, ${ }^{47}$ L. G. Efimov, ${ }^{18}$ M. Elnimr,${ }^{50}$ J. Engelage,${ }^{4}$ G. Eppley, ${ }^{36}$ B. Erazmus, ${ }^{41}$ M. Estienne, ${ }^{41}$ L. Eun,${ }^{31}$ O. Evdokimov ${ }^{8}$ R. Fatemi,${ }^{20}$ J. Fedorisin ${ }^{18}$ R. G. Fersch, ${ }^{20}$ E. Finch,${ }^{52}$ V. Fine, ${ }^{3}$ Y. Fisyak, ${ }^{3}$ C. A. Gagliardi, ${ }^{42}$ D. R. Gangadharan, ${ }^{6}$ M. S. Ganti, ${ }^{47}$ A. Geromitsos, ${ }^{41}$ F. Geurts, ${ }^{36}$ P. Ghosh ${ }^{47}$ Y. N. Gorbunov, ${ }^{9}$ A. Gordon, ${ }^{3}$ O. Grebenyuk, ${ }^{22}$ D. Grosnick ${ }^{46}$ S. M. Guertin, ${ }^{6}$ A. Gupta, ${ }^{17}$ W. Guryn,${ }^{3}$ B. Haag, ${ }^{5}$ A. Hamed, ${ }^{42}$ L-X. Han ${ }^{40}$ J. W. Harris, ${ }^{52}$ J. P. Hays-Wehle, ${ }^{23}$ M. Heinz, ${ }^{52}$ S. Heppelmann, ${ }^{31}$ A. Hirsch, ${ }^{33}$ E. Hjort, ${ }^{22}$ G. W. Hoffmann, ${ }^{43}$ D. J. Hofman, ${ }^{8}$ R. S. Hollis, ${ }^{8}$ B. Huang ${ }^{38}$ H. Z. Huang, ${ }^{6}$ T. J. Humanic, ${ }^{28}$ L. Huo, ${ }^{42}$ G. Igo, ${ }^{6}$ A. Iordanova, ${ }^{8}$ P. Jacobs,${ }^{22}$ W. W. Jacobs,${ }^{15}$ C. Jena, ${ }^{13}$ F. Jin ${ }^{40}$ J. Joseph,${ }^{19}$ E. G. Judd,${ }^{4}$ S. Kabana,${ }^{41}$ K. Kang, ${ }^{44}$ J. Kapitan, ${ }^{11}$ K. Kauder, ${ }^{8}$ D. Keane, ${ }^{19}$ A. Kechechyan, ${ }^{18}$ D. Kettler, ${ }^{49}$ D. P. Kikola, ${ }^{22}$ J. Kiryluk, ${ }^{22}$ A. Kisiel,${ }^{48}$ V. Kizka, ${ }^{18}$ S. R. Klein,${ }^{22}$ A. G. Knospe, ${ }^{52}$ A. Kocoloski, ${ }^{23}$ D. D. Koetke, ${ }^{46}$ T. Kollegger, ${ }^{12}$ J. Konzer, ${ }^{33}$ I. Koralt,${ }^{29}$ L. Koroleva, ${ }^{16}$ W. Korsch, ${ }^{20}$ L. Kotchenda ${ }^{26}$

V. Kouchpil, ${ }^{11}$ P. Kravtsov, ${ }^{26}$ K. Krueger, ${ }^{1}$ M. Krus, ${ }^{10}$ L. Kumar, ${ }^{19}$ P. Kurnadi, ${ }^{6}$ M. A. C. Lamont, ${ }^{3}$ J. M. Landgraf, ${ }^{3}$ S. LaPointe, ${ }^{50}$ J. Lauret, ${ }^{3}$ A. Lebedev,${ }^{3}$ R. Lednicky, ${ }^{18}$ C-H. Lee, ${ }^{34}$ J. H. Lee, ${ }^{3}$ W. Leight, ${ }^{23}$ M. J. LeVine, ${ }^{3}$ C. Li,${ }^{38}$ L. Li ${ }^{43}$ N. Li,${ }^{51}$ W. Li, ${ }^{40}$ X. Li ${ }^{33}$ X. Li, ${ }^{39}$ Y. Li,,${ }^{44}$ Z. M. Li, ${ }^{51}$ M. A. Lisa, ${ }^{28}$ F. Liu, ${ }^{51}$ H. Liu, ${ }^{5}$ J. Liu, ${ }^{66}$ T. Ljubicic, ${ }^{3}$ W. J. Llope,,${ }^{36}$ R. S. Longacre, ${ }^{3}$ W. A. Love, ${ }^{3}$ Y. Lu, ${ }^{38}$ E. V. Lukashov, ${ }^{26}$ X. Luo, ${ }^{38}$ G. L. Ma ${ }^{40}$ Y. G. Ma ${ }^{40}$ D. P. Mahapatra, ${ }^{13}$ R. Majka, ${ }^{52}$ O. I.

Mall,${ }^{5}$ L. K. Mangotra, ${ }^{17}$ R. Manweiler ${ }^{46}$ S. Margetis, ${ }^{19}$ C. Markert,${ }^{43}$ H. Masui, ${ }^{22}$ H. S. Matis, ${ }^{22}$ Yu. A. Matulenko, ${ }^{32}$ D. McDonald, ${ }^{36}$ T. S. McShane, ${ }^{9}$ A. Meschanin, ${ }^{32}$ R. Milner, ${ }^{23}$ N. G. Minaev,${ }^{32}$ S. Mioduszewski,${ }^{42}$ M. K. Mitrovski, ${ }^{12}$ B. Mohanty, ${ }^{47}$ M. M. Mondal,${ }^{47}$ B. Morozov,${ }^{16}$ D. A. Morozov,${ }^{32}$ M. G. Munhoz,${ }^{37}$ M. Naglis, ${ }^{22}$ B. K. Nandi,${ }^{14}$ T. K. Nayak ${ }^{47}$ P. K. Netrakanti, ${ }^{33}$ M. J. Ng, ${ }^{4}$ L. V. Nogach, ${ }^{32}$ S. B. Nurushev, ${ }^{32}$ G. Odyniec, ${ }^{22}$ A. Ogawa, ${ }^{3}$ A. Ohlson, ${ }^{52}$ V. Okorokov, ${ }^{26}$ E. W. Oldag, ${ }^{43}$ D. Olson, ${ }^{22}$ M. Pachr, ${ }^{10}$ B. S. Page, ${ }^{15}$ S. K. Pal,${ }^{47}$ Y. Pandit, ${ }^{19}$ Y. Panebratsev, ${ }^{18}$ T. Pawlak, ${ }^{48}$ T. Peitzmann, ${ }^{27}$

C. Perkins, ${ }^{4}$ W. Peryt,${ }^{48}$ S. C. Phatak, ${ }^{13}$ P. Pile, ${ }^{3}$ M. Planinic,,${ }^{53}$ M. A. Ploskon, ${ }^{22}$ J. Pluta, ${ }^{48}$ D. Plyku, ${ }^{29}$ N. Poljak, ${ }^{53}$ A. M.

Poskanzer, ${ }^{22}$ B. V. K. S. Potukuchi, ${ }^{17}$ C. B. Powell, ${ }^{22}$ D. Prindle,${ }^{49}$ C. Pruneau,${ }^{50}$ N. K. Pruthi,${ }^{30}$ P. R. Pujahari, ${ }^{14}$ J. Putschke, ${ }^{52}$ H. Qiu, ${ }^{21}$ R. Raniwala, ${ }^{35}$ S. Raniwala, ${ }^{35}$ R. L. Ray, ${ }^{43}$ R. Redwine, ${ }^{23}$ R. Reed,${ }^{5}$ H. G. Ritter, ${ }^{22}$ J. B. Roberts, ${ }^{36}$ O. V. Rogachevskiy, ${ }^{18}$ J. L. Romero, ${ }^{5}$ A. Rose,${ }^{22}$ L. Ruan, ${ }^{3}$ S. Sakai, ${ }^{6}$ I. Sakrejda, ${ }^{22}$ T. Sakuma, ${ }^{23}$ S. Salur, ${ }^{5}$ J. Sandweiss, ${ }^{52}$ E. Sangaline, ${ }^{5}$ J. Schambach, ${ }^{43}$ R. P. Scharenberg, ${ }^{33}$ A. M. Schmah, ${ }^{22}$ N. Schmitz,${ }^{24}$ T. R. Schuster, ${ }^{12}$ J. Seele, ${ }^{23}$ J. Seger, ${ }^{9}$ I. Selyuzhenkov, ${ }^{15}$ P. Seyboth, ${ }^{24}$ E. Shahaliev, ${ }^{18}$ M. Shao, ${ }^{38}$ M. Sharma, ${ }^{50}$ S. S. Shi, ${ }^{51}$ E. P. Sichtermann, ${ }^{22}$ F. Simon, ${ }^{24}$ R. N. Singaraju, ${ }^{47}$ M. J. Skoby, ${ }^{33}$ N. Smirnov, ${ }^{52}$ P. Sorensen, ${ }^{3}$ H. M. Spinka, ${ }^{1}$ B. Srivastava, ${ }^{33}$ T. D. S. Stanislaus, ${ }^{46}$ D. Staszak, ${ }^{6}$ J. R. Stevens, ${ }^{15}$ R. Stock,${ }^{12}$ M. Strikhanov, ${ }^{26}$ B. Stringfellow, ${ }^{33}$ A. A. P. Suaide,${ }^{37}$ M. C. Suarez,${ }^{8}$ N. L. Subba, ${ }^{19}$ M. Sumbera ${ }^{11}$ X. M. Sun, ${ }^{22}$ Y. Sun,${ }^{38}$ Z. Sun, ${ }^{21}$ B. Surrow ${ }^{23}$ D. N. Svirida, ${ }^{16}$ T. J. M. Symons,${ }^{22}$ A. Szanto de Toledo, ${ }^{37}$ J. Takahashi, ${ }^{7}$ A. H. Tang, ${ }^{3}$ Z. Tang, ${ }^{38}$ L. H. Tarini, ${ }^{50}$ T. Tarnowsky, ${ }^{25}$ D. Thein, ${ }^{43}$ J. H. Thomas, ${ }^{22}$ J. Tian, ${ }^{40}$ A. R. Timmins,${ }^{50}$ S. Timoshenko, ${ }^{26}$

D. Tlusty, ${ }^{11}$ M. Tokarev, ${ }^{18}$ V. N. Tram,${ }^{22}$ S. Trentalange,${ }^{6}$ R. E. Tribble,${ }^{42}$ O. D. Tsai,${ }^{6}$ T. Ullrich,${ }^{3}$ D. G. Underwood,${ }^{1}$ G. Van

Buren, ${ }^{3}$ M. van Leeuwen, ${ }^{27}$ G. van Nieuwenhuizen, ${ }^{23}$ J. A. Vanfossen Jr., ${ }^{19}$ R. Varma,${ }^{14}$ G. M. S. Vasconcelos, ${ }^{7}$ A. N.

Vasiliev ${ }^{32}$ F. Videbæk, ${ }^{3}$ Y. P. Viyogi, ${ }^{47}$ S. Vokal, ${ }^{18}$ S. A. Voloshin,${ }^{50}$ M. Wada, ${ }^{43}$ M. Walker, ${ }^{23}$ F. Wang, ${ }^{33}$ G. Wang, ${ }^{6}$

H. Wang, ${ }^{25}$ J. S. Wang, ${ }^{21}$ Q. Wang, ${ }^{33}$ X. L. Wang, ${ }^{38}$ Y. Wang, ${ }^{44}$ G. Webb,${ }^{20}$ J. C. Webb, ${ }^{3}$ G. D. Westfall, ${ }^{25}$ C. Whitten Jr., ${ }^{6}$

H. Wieman, ${ }^{22}$ S. W. Wissink, ${ }^{15}$ R. Witt, ${ }^{45}$ Y. F. Wu ${ }^{51}$ W. Xie, ${ }^{33}$ H. Xu, ${ }^{21}$ N. Xu ${ }^{22}$ Q. H. Xu ${ }^{39}$ W. Xu, ${ }^{6}$ Y. Xu, ${ }^{38}$ Z. Xu, ${ }^{3}$

L. Xue, ${ }^{40}$ Y. Yang, ${ }^{21}$ P. Yepes, ${ }^{36}$ K. Yip, ${ }^{3}$ I-K. Yoo, ${ }^{34}$ Q. Yue, ${ }^{44}$ M. Zawisza, ${ }^{48}$ H. Zbroszczyk, ${ }^{48}$ W. Zhan, ${ }^{21}$ J. B. Zhang, ${ }^{51}$ S. Zhang, ${ }^{40}$ W. M. Zhang, ${ }^{19}$ X. P. Zhang, ${ }^{44}$ Y. Zhang, ${ }^{22}$ Z. P. Zhang, ${ }^{38}$ J. Zhao,${ }^{40}$ C. Zhong, ${ }^{40}$ W. Zhou, ${ }^{39}$ X. Zhu, ${ }^{44}$ Y. H. Zhu ${ }^{40}$ R. Zoulkarneev, ${ }^{18}$ and Y. Zoulkarneeva ${ }^{18}$

(STAR Collaboration)

${ }^{1}$ Argonne National Laboratory, Argonne, Illinois 60439, USA

${ }^{2}$ University of Birmingham, Birmingham, United Kingdom

${ }^{3}$ Brookhaven National Laboratory, Upton, New York 11973, USA

${ }^{4}$ University of California, Berkeley, California 94720, USA

${ }^{5}$ University of California, Davis, California 95616, USA

${ }^{6}$ University of California, Los Angeles, California 90095, USA

${ }^{7}$ Universidade Estadual de Campinas, Sao Paulo, Brazil

${ }^{8}$ University of Illinois at Chicago, Chicago, Illinois 60607, USA

${ }^{9}$ Creighton University, Omaha, Nebraska 68178, USA

${ }^{10}$ Czech Technical University in Prague, FNSPE, Prague, CZ-115 19, Czech Republic 


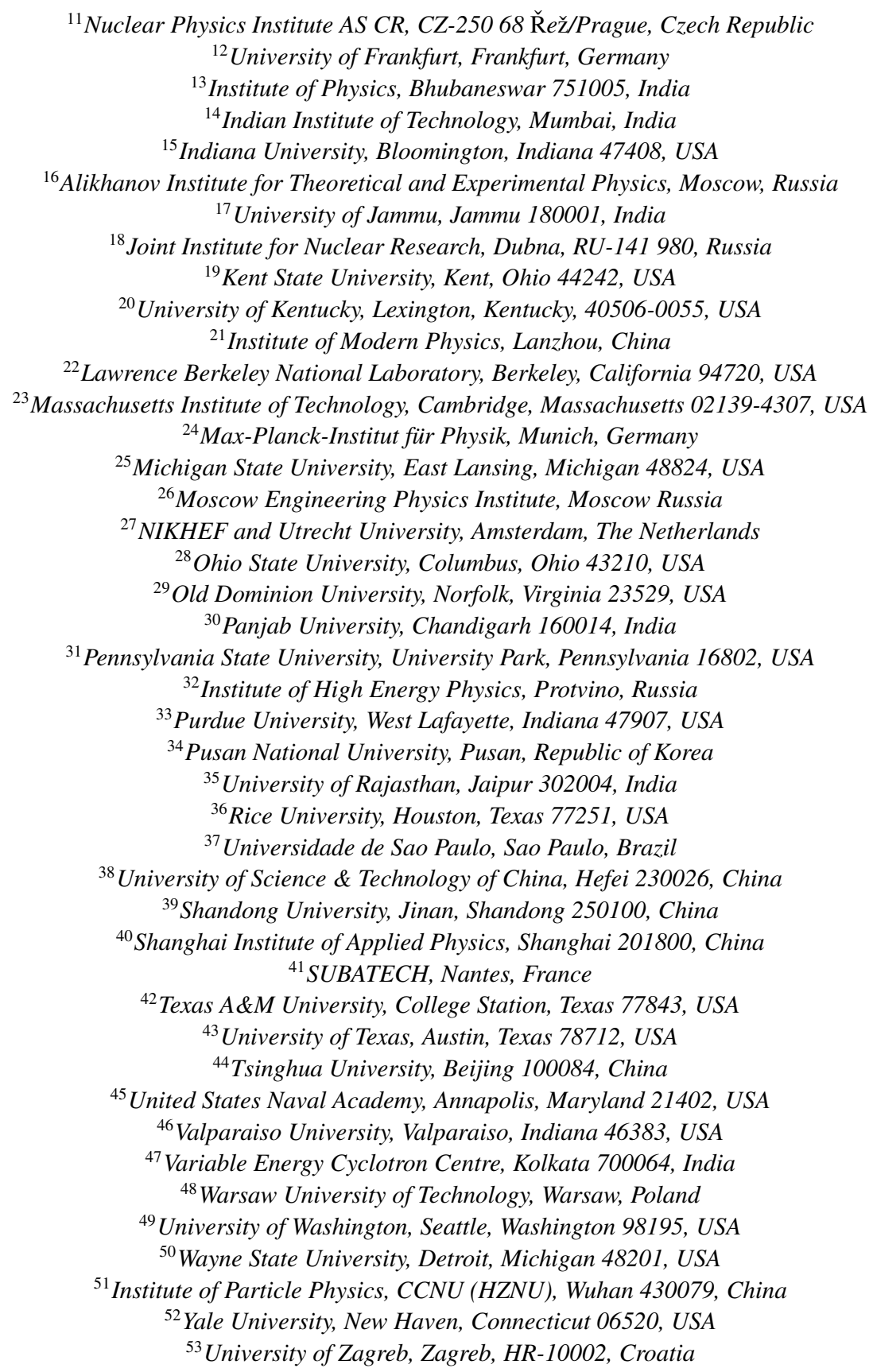

(Received 18 August 2010; published 28 March 2011)

Identified charged pion, kaon, and proton spectra are used to explore the system size dependence of bulk freezeout properties in $\mathrm{Cu}+\mathrm{Cu}$ collisions at $\sqrt{s_{N N}}=200$ and $62.4 \mathrm{GeV}$. The data are studied with hydrodynamically motivated blast-wave and statistical model frameworks in order to characterize the freeze-out properties of the system. The dependence of freeze-out parameters on beam energy and collision centrality is discussed. Using the existing results from $\mathrm{Au}+\mathrm{Au}$ and $\mathrm{pp}$ collisions, the dependence of freeze-out parameters on the system size is also explored. This multidimensional systematic study furthers our understanding of the QCD phase diagram revealing the importance of the initial geometrical overlap of the colliding ions. The analysis of $\mathrm{Cu}+\mathrm{Cu}$ collisions expands the system size dependence studies from $\mathrm{Au}+\mathrm{Au}$ data with detailed measurements in the smaller system. The systematic trends of the bulk freeze-out properties of charged particles is studied with respect to the total charged particle multiplicity at midrapidity, exploring the influence of initial state effects.

DOI: 10.1103/PhysRevC.83.034910

PACS number(s): 25.75.-q 


\section{INTRODUCTION}

An experimental study of relativistic heavy-ion collisions augments our understanding of the QCD phase diagram [1]. The high energy density reached in such collisions at the Relativistic Heavy Ion Collider (RHIC) is believed to result in a novel state of hot and dense matter with properties strikingly different from that of a hadron gas or ordinary nuclear matter [2].

The bulk properties of particle production are studied using identified particle spectra at low momentum. Model-dependent interpretations of the measured data provide insight into the complex dynamics of the collision and further explore the QCD phases through which the collision evolves. The dense system formed in the early stages of the collision continuously expands and cools, until kinetic freeze-out, beyond which the particles stream freely into the detector. Through measurements of species abundances and transverse momentum distributions, information about the final stages of the collision evolution at chemical and kinetic freeze-out can be inferred.

The relative particle abundances and spectral shapes discussed here were tested within the frameworks of statistical (chemical-equilibrium) [3] and blast-wave [4] models. In the chemical-equilibrium model, particle abundances relative to the total system volume (assumed to be the same for all particle species) are described by the system temperature at freeze-out, the baryon and strangeness chemical potentials, and the strangeness suppression factor. The blast-wave model describes spectral shapes assuming a locally thermalized source with a common transverse flow velocity field. The results from $\mathrm{Au}+\mathrm{Au}$ collisions at $\sqrt{s_{N N}}=200$ and at $62.4 \mathrm{GeV}$ [5-7] have shown that the chemical freeze-out temperature $T_{\mathrm{ch}}$ has little dependence on centrality whereas the kinetic freeze-out temperature $T_{\text {kin }}$ decreases with increasing centrality of the collision. Furthermore, the radial flow velocity $\beta$ increases with increasing centrality. The observed changes in $T_{\text {kin }}$ and $\beta$ with centrality are consistent with higher energy and pressure in the initial state for more central events. On the other hand, the centrality independence of the extracted chemical freeze-out temperature indicates that, even for different initial conditions, collisions always evolve to the same chemical freeze-out. Moreover, the value for the chemical freeze-out temperature in $\mathrm{Au}+\mathrm{Au}$ is close to the critical temperature predicted by some lattice QCD calculations [8]. This suggests that chemical freeze-out coincides with hadronization and, therefore, $T_{\mathrm{ch}}$ provides a lower-limit estimate for the temperature of the prehadronic state [9]. The systematic behavior of the kinetic freeze-out properties with charged hadron multiplicity appears to follow the same trend for all energies and systems at RHIC $[5,6]$. In this paper, the systematic studies of the QCD phase diagram from heavy-ion collisions are enriched by the addition of new RHIC data from $\mathrm{Cu}+\mathrm{Cu}$ collisions at $\sqrt{s_{N N}}=200$ and $62.4 \mathrm{GeV}$.

\section{THE STAR EXPERIMENT}

The $\mathrm{Cu}+\mathrm{Cu}$ data presented here were collected by the STAR experiment during the RHIC 2005 run. Copper nuclei $\left({ }^{63} \mathrm{Cu}\right)$ were collided at $\sqrt{s_{N N}}=200$ and $62.4 \mathrm{GeV}$. Data were recorded with a minimum bias trigger obtained from the beambeam Cherenkov counters [10] coupled with information from the zero-degree calorimeters [11]. This trigger is found to be sensitive to the top $\sim 85 \%$ of the inelastic cross section. The data studied here correspond to the top $60 \%$ of the inelastic cross section (minimum bias) where little or no inefficiency of the triggering or vertex reconstruction is found. These 0\%-60\% minimum-bias events, with $24 \mathrm{M}$ and $10 \mathrm{M}$ events recorded at 200 and $62.4 \mathrm{GeV}$, respectively, were divided into six centrality bins, each corresponding to a $10 \%$ interval of the geometric cross section. Transverse mass distributions for charged pions, charged kaons, protons, and antiprotons, previously reported by STAR for $\sqrt{s_{N N}}=200 \mathrm{GeV} p p$ collisions and $\mathrm{Au}+\mathrm{Au}$ collisions at 62.4 and $200 \mathrm{GeV}$ [5-7], are used for comparison.

The STAR time projection chamber (TPC) [12] tracks particle trajectories over a wide range of momentum at midrapidity $(|\eta|<1.8)$. The particle identification at low $p_{T}$ uses measurements of truncated mean ionization energy loss $\langle d E / d x\rangle$ of the charged particles traversing the TPC. Particles of different mass show distinct patterns in the $\langle d E / d x\rangle$ dependence, as shown in Fig. 1, left panel. This allows statistical separation of pions and kaons in the momentum range $0.25<p_{T}<0.80 \mathrm{GeV} / c$ at midrapidity $(|y|<0.1)$ and of protons and antiprotons from other species in the range $0.40<p_{T}<1.20 \mathrm{GeV} / c$.

The momentum measurement is given by the curvature of the particle trajectories as they pass through the $0.5 \mathrm{~T}$ magnetic field of the STAR detector. To ensure optimal $d E / d x$ resolution, only primary tracks, with $d c a$ (distance of closest approach between the particle trajectory and the event vertex) less than $3 \mathrm{~cm}$, and at least 25 out of 45 possible fit points are used in this analysis. Particle identification at midrapidity $(|y|<0.1)$ is achieved by fits to the $Z$ variable, defined as a logarithm of $\langle d E / d x\rangle$ divided by the theoretically expected value for each particle type, given by Bethe-Bloch formula [13]. This new variable is introduced to remove the strong $p_{T}$ dependence at low momenta. Such a normalized distribution is created for a given particle and centrality and is divided into narrow transverse momentum slices (width $\Delta p_{T}=$ $50 \mathrm{MeV} / c$ ). These momentum projections are fit with a combined four-Gaussian function, one for each of the particle species of a given charge: $\pi, \mathrm{K}, p$, and $e$. The integral of each Gaussian provides the raw yield at each momentum. This procedure is repeated for each particle species in order to assign the correct rapidity for each track, using the mass of the particle. Thus, fits to the auxiliary particles in each distribution (for example $\mathrm{K}, p$, and $e$ for $\pi$ analysis) are used only to estimate the contamination when bands overlap. The right panel of Fig. 1 shows an example for pion-yield extraction for one momentum slice. For more details see Ref. [7].

The raw yields extracted from each of the four-Gaussian fits are then corrected for detector acceptance, single-track reconstruction efficiencies, and other effects as discussed below. To determine the correction factors, simulated tracks were embedded into real data on the raw signal level and run through the standard reconstruction chain. The estimated 

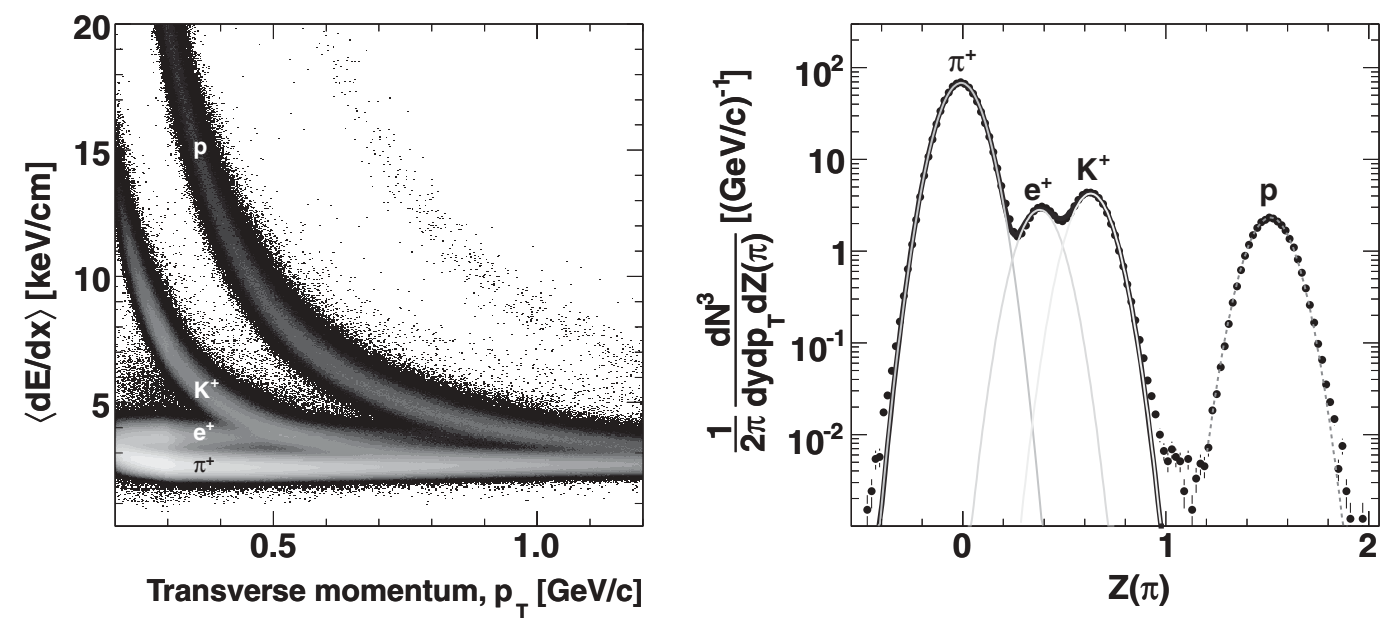

FIG. 1. The left panel shows the truncated mean ionization energy loss $(\langle d E / d x\rangle)$ in the TPC as a function of transverse momentum for positively charged tracks from $200 \mathrm{GeV} \mathrm{Cu}+\mathrm{Cu}$ collisions. The right panel shows $Z(\pi)$, the logarithm of the measured $\langle d E / d x\rangle$ divided by the theoretical expectation for energy loss of charged pions, for $0.40<p_{T}<0.45 \mathrm{GeV} / c$. Also shown is an example four-Gaussian fit that is used to extract the raw yields for different species.

single-track reconstruction efficiency is about $80 \%$ for $\pi^{ \pm}$ in $\mathrm{Cu}+\mathrm{Cu}$ collisions and exhibits a small centrality and $p_{T}$ dependence. The $p_{T}$ spectrum has also been corrected for the energy loss due to multiple scattering, which affects the reconstructed momentum at low values. The maximum value of this mass-dependent correction to the measured $p_{T}$ value for $\mathrm{K}^{ \pm}$and $p(\bar{p})$ was found to be $2 \%$ and $3 \%$, respectively, for the lowest measured $p_{T}$ bin. An additional correction for the background contamination in the proton sample is made. The background protons arise predominantly from secondary interactions in the beam pipe and detector material (knock-out protons). It is estimated from data to be about $40 \%$ at $p_{T}=400 \mathrm{MeV} / c$, diminishing to near zero at $p_{T}=$ $1 \mathrm{GeV} / c$, as shown in Fig. 2. To estimate this correction factor we compare the distribution of proton $d c a$ to that of the antiprotons (see Ref. [7] for more details). The measured antiprotons do not have a contribution from secondary interactions, so their $d c a$ distribution is unaffected by this background. As such, comparing the antiproton $d c a$ distribution to that of the proton can provide a measure for this background. To calculate the correction, two steps are needed. First, the antiprotons are scaled (divided) by the measured raw $\bar{p} / p$ ratio $\bar{p} / p \sim$ 0.83 and measured at (lowest-background) higher-momenta $\left(p_{T}>0.9 \mathrm{GeV} / c\right)$ before other corrections are applied. The difference in the two $d c a$ distributions is used to estimate the shape of the background contribution. In the second step, the proton $d c a$ distribution is fit using a sum of the antiproton $d c a$ distribution and an analytical representation of background shape both scaled by independent factors determined in the fit. Pion yields are additionally corrected for feed-down contributions from weakly decaying particles, muon contamination, and background pions from detector material. This correction is found to decrease from about $15 \%$ at $0.3 \mathrm{GeV} / c$ to about $5 \%$ at $1 \mathrm{GeV} / c$. The (anti)protons presented in this paper are inclusive measurements (not corrected for weak decays). It has been found in previous studies that the analysis cuts used for the low $p_{T}$ identified proton studies $(d c a<3 \mathrm{~cm})$ reject only a negligible fraction of daughter protons from the hyperon decays [5]. Therefore, our sample reflects the total baryon production in the collision. Earlier Au + Au studies [5] and preliminary Lambda-hyperon spectra from $\mathrm{Cu}+\mathrm{Cu}$ collisions [14] indicate that the freezeout spectral shapes are similar for $\Lambda \mathrm{s}$ and protons, resulting in similar-shaped spectra for primary and feed-down protons. The fraction of the weak-decay feed-down protons is estimated to be about $30 \%$ [15].

This analysis technique is used to obtain the low- $p_{T}$ particle spectra for all centrality bins at both 200 and $62.4 \mathrm{GeV}$ center-of-mass energies and for the $\mathrm{Cu}+\mathrm{Cu}$ and $\mathrm{Au}+\mathrm{Au}$ colliding systems. Additional technical details on the analysis and applied corrections can be found in Refs. [5,6] with a thorough overview in Ref. [7].

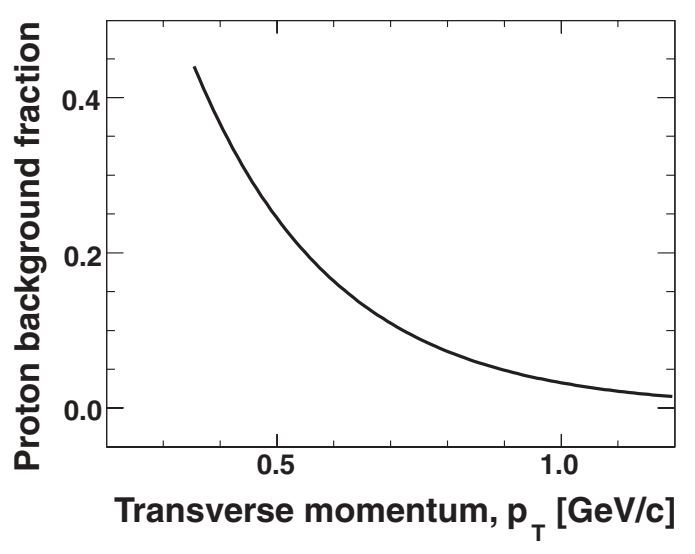

FIG. 2. Estimated fraction of background protons in the raw proton sample as function of transverse momentum, for the most central $\sqrt{s_{N N}}=200 \mathrm{GeV} \mathrm{Cu}+\mathrm{Cu}$ collisions. No strong centrality or energy dependence for this correction was observed for all $\mathrm{Cu}+\mathrm{Cu}$ data available. 


\section{RESULTS}

The transverse momentum spectra are shown in Figs. 3 and 4 for $\pi^{ \pm}$(leftmost column), $\mathrm{K}^{ \pm}$(center), and (anti)protons (right) in $\mathrm{Cu}+\mathrm{Cu}$ collisions. The top row presents the data for $\sqrt{s_{N N}}=200 \mathrm{GeV}$, whilst data for $62.4 \mathrm{GeV}$ are shown in the bottom row. The symbol shades represent different centrality bins. The particle and antiparticle spectral shapes are similar for all species in each centrality bin. At both collision energies a mass dependence is observed in the slope of the particle spectra. Due to the large number of events recorded and good tracking efficiency, the statistical errors are less than $1 \%$. The systematic uncertainties are similar to those determined in prior analyses of low- $p_{T}$ spectra in $\mathrm{Au}+\mathrm{Au}$ collisions [7]. Systematic errors are divided into two classes: point-topoint and scale uncertainties. The overall scale uncertainty, mostly due to the embedding procedure for the single-track reconstruction efficiency, is estimated to be $5 \%$ for all particle species. Point-to-point uncertainties are determined for each $p_{T}$ bin and particle species. For pions and kaons, this error is evaluated to be less than $7 \%$ and $13 \%$, respectively. These maximal errors represent $p_{T}$ bins where a significant $\langle d E / d x\rangle$ overlap occurs between $\pi^{ \pm}, \mathrm{K}^{ \pm}$, or $e^{ \pm}$. For protons and antiprotons, the maximum error is $5 \%$. At low $p_{T}$, the proton uncertainty is greater than that for antiprotons (4.0\% versus $1.3 \%$, respectively, at $p_{T}=400-450 \mathrm{MeV} / c$ ) owing to the additional uncertainty from the proton's background. The uncertainty due to the background decreases rapidly from $3.7 \%$ at $p_{T}=400-450 \mathrm{MeV} / c$ to $1.5 \%$ for $p_{T}>1 \mathrm{GeV} / c$.

For the antiparticle to particle yield ratios, systematic errors are much reduced due to a cancellation of the efficiency uncertainties and a partial cancellation of extrapolation uncertainties, as described above. A systematic uncertainty of $2 \%, 3 \%$, and $5 \%$ is assigned to $\pi^{-} / \pi^{+}, \mathrm{K}^{-} / \mathrm{K}^{+}$, and $\bar{p} / p$, respectively.

We further fit the obtained $p_{T}$ distributions to extract system properties at different stages of the collision evolution. The first fit to the data probes collision properties at kinetic freeze-out. Here, a blast-wave model [4] is used to simultaneously fit the $\pi^{ \pm}, \mathrm{K}^{ \pm}$, and (anti)proton spectra at a given centrality. This fit provides a good description of the spectral shapes, as illustrated in Fig. 5 with results from most central $200-\mathrm{GeV} \mathrm{Cu}+\mathrm{Cu}$ data. The $\pi^{ \pm}$data points for $p_{T}<0.5 \mathrm{GeV} / c$ are excluded from the blast-wave fits to reduce the effects of resonance-decay contributions as done in previous works [5-7]. Including this low- $p_{T}$ region in the fit leads to a poorer description of proton and kaon shapes; however, the resultant modification of the extracted parameters remains well within their systematic uncertainty. The freezeout parameters obtained from this model are discussed later. Also shown in Fig. 5 are Bose-Einstein $\left[\propto 1 /\left(\exp \frac{m_{T}}{T}-1\right)\right]$ fits to the $\pi^{ \pm}$, which provide a slightly better description of these data. For evaluation of the systematic uncertainties from extrapolation, $m_{T}$ exponential $\left(\propto 1 / \exp \frac{m_{T}}{T}\right)$ and Boltzmann $\left(\propto m_{T} / \exp \frac{m_{T}}{T}\right)$ fits are also used in the analysis (for more details see Ref. [7]).

The particle mean- $p_{T}$ and total particle yields at midrapidity $(|y|<0.1)$ are shown in Figs. 6 and 7, respectively. The values presented for kaons and (anti)protons are determined from the measured spectral points, extrapolated outside the fiducial range using blast-wave fits discussed above. Similarly, a combination of the measured data points and extrapolation from Bose-Einstein fits is used for the pions. The measured

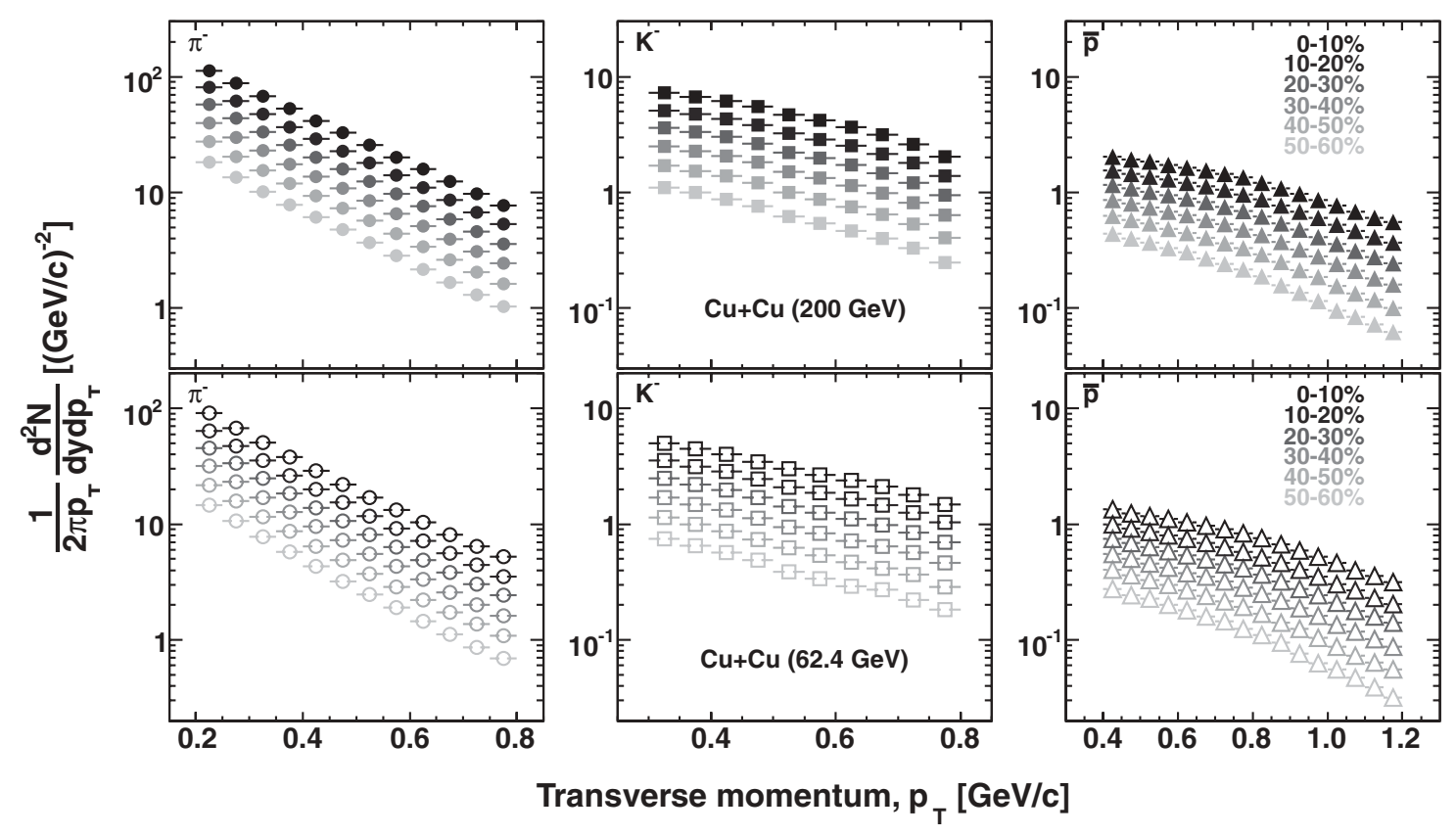

FIG. 3. The top row shows negatively charged pion (leftmost column), kaon (center), and antiproton (right) spectra from $\mathrm{Cu}+\mathrm{Cu}$ collisions at $\sqrt{s_{N N}}=200 \mathrm{GeV}$. Six centrality classes are shown as dark (central $\left.0 \%-10 \%\right)$ to light $(50 \%-60 \%)$ shades. Spectra for $62.4 \mathrm{GeV} \mathrm{Cu}+\mathrm{Cu}$ are shown on the bottom row. Statistical and systematic errors [which do not exceed $7 \%\left(\pi^{-}, \bar{p}\right)$ and $\left.13 \%\left(K^{-}\right)\right]$are smaller than the symbol size. 


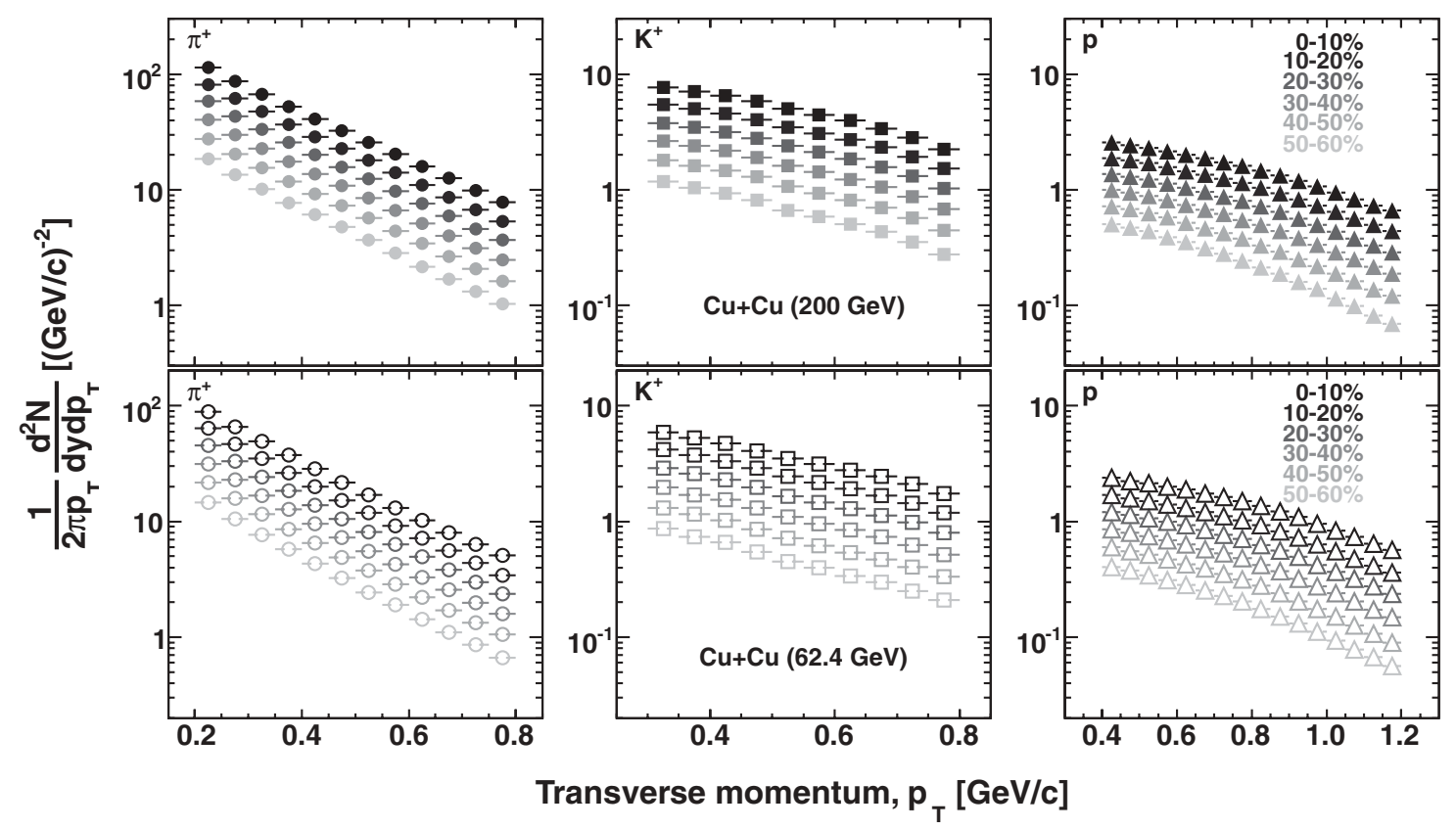

FIG. 4. The top row shows positively charged pion (leftmost column), kaon (center), and proton (right) spectra from $\mathrm{Cu}+\mathrm{Cu}$ collisions at $\sqrt{s_{N N}}=200 \mathrm{GeV}$. Six centrality classes are shown as dark (central $\left.0 \%-10 \%\right)$ to light $(50 \%-60 \%)$ shades. Spectra for $62.4 \mathrm{GeV} \mathrm{Cu}+$ $\mathrm{Cu}$ are shown on the bottom row. Statistical and systematic errors [which do not exceed $7 \%\left(\pi^{+}, p\right)$ and $13 \%\left(K^{+}\right)$] are smaller than the symbol size.

fraction of the total yield is found to be $62 \%$ for $\pi^{ \pm}, 58 \%$ for $\mathrm{K}^{ \pm}$, and $65 \%$ for (anti)protons for the most central $200 \mathrm{GeV}$ data; these fractions are slightly higher in other centrality bins and at lower energy [16]. The systematic uncertainty in $d N / d y$ and mean $p_{T}$, shown in the figures, includes the extrapolation uncertainty evaluated by means of the various model fits mentioned earlier. Overall, these are estimated to be near $15 \%$ of the yields outside the fiducial range for pions and kaons and $15 \%-25 \%$ of the extrapolated yields for protons and antiprotons, depending on centrality.

We also determine the total charged hadron production per unit of pseudorapidity, $d N_{\mathrm{ch}} / d \eta$, at midrapidity. The total particle yield at midrapidity for each species, obtained by extrapolating the fits to the measured spectrum in the momentum range outside our fiducial coverage, was corrected for the Jacobian transformation $d N_{\mathrm{ch}} / d y \rightarrow d N_{\mathrm{ch}} / d \eta$. The sum of the total charged pion, kaon, and (anti)proton yields was then corrected for the feed-down of weakly decaying neutral strange particles, providing the estimate of primordial charged hadron yield at midrapidity. A complementary method was also used, integrating over the charged hadron spectra corrected for efficiency, feed-down, and the Jacobian transformation, and yielded consistent results.

The mean $p_{T}$ of each particle species $(\pi, \mathrm{K}, p)$ increases with the number of charged hadrons at midrapidity $d N_{\mathrm{ch}} / d \eta$, as shown in Fig. 6. Moreover, the mean $p_{T}$ for each particle species appears to scale with $d N_{\mathrm{ch}} / d \eta$ at midrapidity, and to be independent of the colliding system and the center-of-mass energy. The particle yields show the same systematic scaling features with $d N_{\mathrm{ch}} / d \eta$ as mean $p_{T}$ across system and collision energy; see Fig. 7. In this logarithmic representation, the particle yields for each species appear to increase linearly with multiplicity, with $\mathrm{Cu}+\mathrm{Cu}$ matching the $\mathrm{Au}+\mathrm{Au}$ data at similar values of $d N_{\mathrm{ch}} / d \eta$. When shown on a linear scale, the integrated yields exhibit a near-linear dependence with $d N_{\mathrm{ch}} / d \eta$. The logarithmic scale for both axes used here preserves the apparent linear dependencies whilst better illustrating the lower-multiplicity $\mathrm{Cu}+\mathrm{Cu}$ data. For the detailed features we investigate the relative particle production in the following.

The relative abundances of particles provide an important insight into the chemical properties of the system. The relative kaon yield reflects the strangeness production in the collision, whereas proton with respect to pion production is dependent on the baryon production and transport. Figure 8(a) shows the ratios for the negatively charged particles, $\bar{p} / \pi^{-}$and $\mathrm{K}^{-} / \pi^{-}$, as a function of $d N_{\mathrm{ch}} / d \eta$, which exhibit similar $d N_{\mathrm{ch}} / d \eta$ scaling behavior at each collision energy. The slight decrease of the values for both ratios seen at the lower collision energy of $62.4 \mathrm{GeV}$ is insignificant within experimental uncertainties. Figure 8 (b) shows the ratios for positively charged particles, $p / \pi^{+}$and $\mathrm{K}^{+} / \pi^{+}$, which also exhibit a $d N_{\mathrm{ch}} / d \eta$-scaling behavior within the same collision energy. The beam-energy effect is reversed here as compared to the ratio of negatively charged particles. Summing over the two charges [Fig. 8(c)], the corresponding ratios exhibit a common scaling behavior with $d N_{\mathrm{ch}} / d \eta$, independent of colliding system and collision energy. The energy dependence of the positive and negative particle ratios, considered separately, points to the effects of baryon transport to midrapidity, which decreases with increasing energy (see also Ref. [16]). 

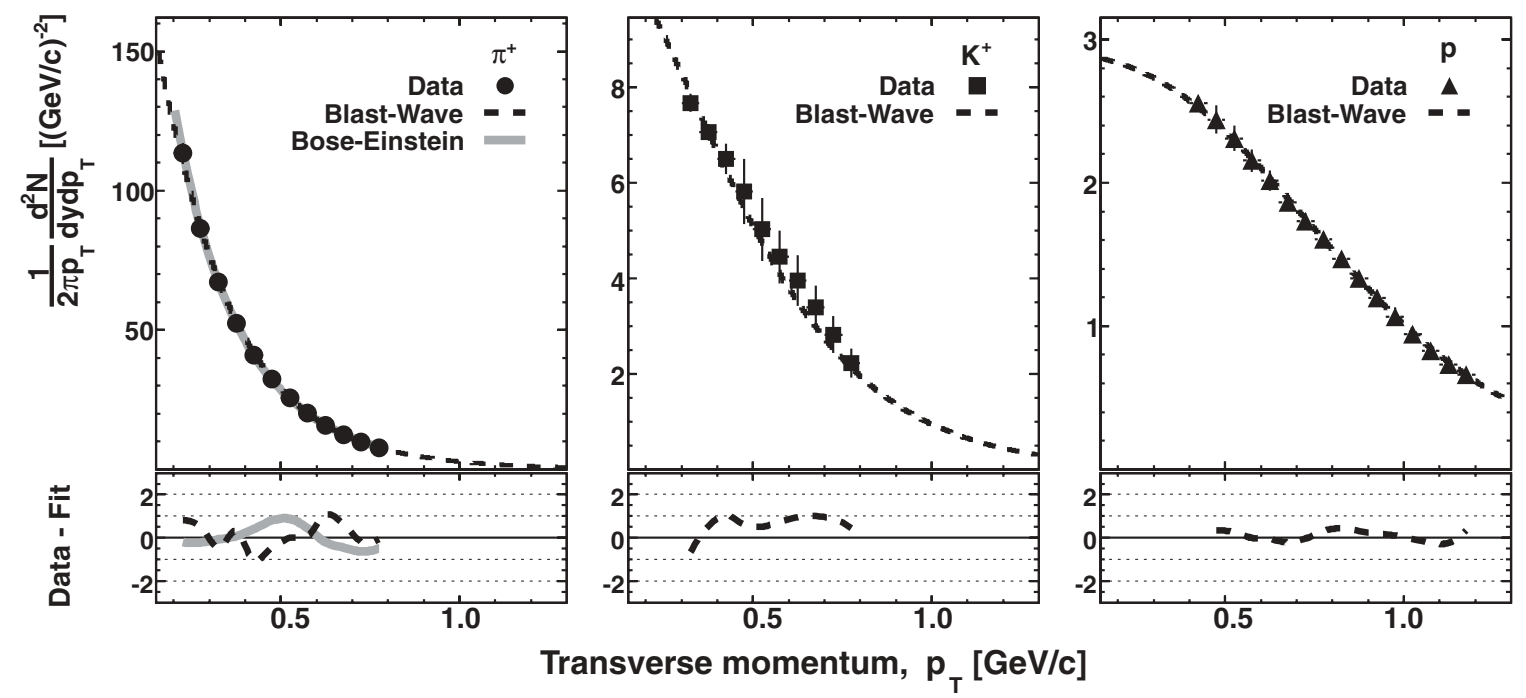

FIG. 5. Comparison between the $10 \%$ most central $\mathrm{Cu}+\mathrm{Cu}$ collision data (symbols) at $\sqrt{s_{N N}}=200 \mathrm{GeV}$ and the corresponding blastwave-model fit (dashed line) to $\pi^{+}$(left), $\mathrm{K}^{+}$(center), and proton (right) spectra-note that the fit is performed simultaneously across species. The pion data points below $p_{T}=0.5 \mathrm{GeV} / c$ were not included in the blast-wave fits to reduce the effect of resonance decays. A Bose-Einstein fit to the pion spectra over the entire fiducial range is also shown. The lower panels illustrate the quality of the fits by showing the difference between the measured points and the fit expressed as the number of standard deviations.

We further explore the kaon production in $\mathrm{Cu}+\mathrm{Cu}$ collisions to gain better insight into production of strange quarks. The $\mathrm{K}^{-} / \pi^{-}$ratio scales with $d N_{\mathrm{ch}} / d \eta$ at both energies and there is no hint of an additional strangeness enhancement of charged kaons in the smaller $\mathrm{Cu}+\mathrm{Cu}$ system compared to the larger $\mathrm{Au}+\mathrm{Au}$ system. Early works from Super Proton Synchrotron (SPS) energies reported such additional relative strangeness enhancement in the $\mathrm{K} / \pi$ ratio for smaller systems, although no final confirmation of this observation is available $[17,18]$. The pion and kaon enhancement factors are compared in Fig. 9. This factor is defined as the yield per mean number

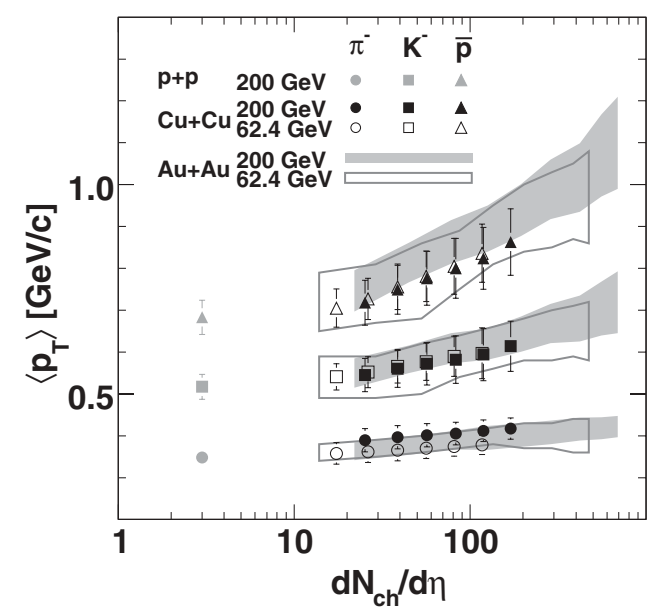

FIG. 6. Mean transverse momentum as a function of charged hadron multiplicity at midrapidity for pions, kaons, and antiprotons. For comparison, the mean- $p_{T}$ values for $\mathrm{Au}+\mathrm{Au}$ data are shown as bands. Filled (open) symbols or bands depict data at $\sqrt{s_{N N}}=$ $200 \mathrm{GeV}(62.4 \mathrm{GeV})$. Error bars represent statistical and systematic uncertainties added in quadrature. of participating nucleons $N_{\text {part }}$ (estimated using a Glauber model) in heavy-ion collisions divided by the respective value in $p p$ collisions. A progressive enhancement of kaon production with respect to pions as a function of collision

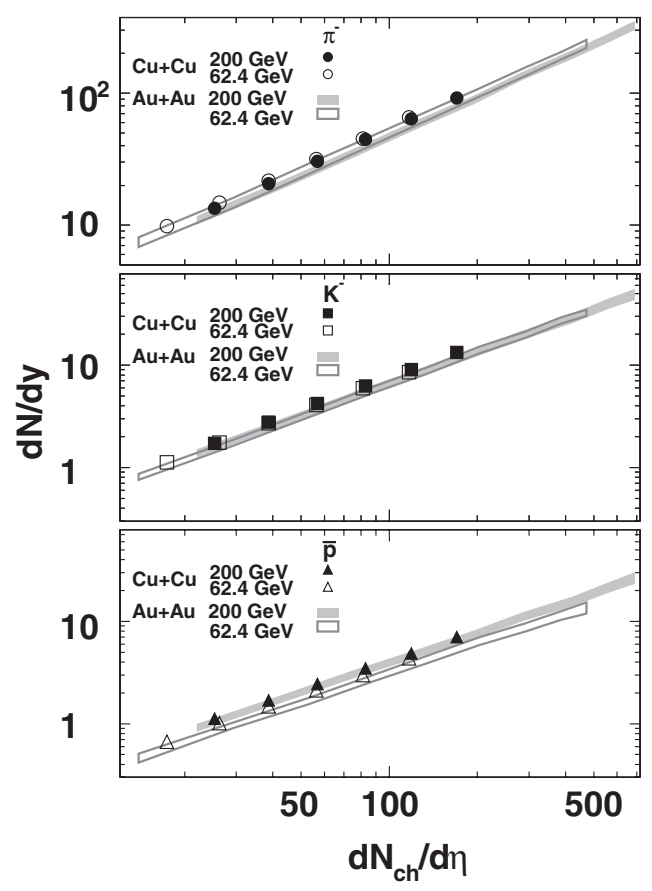

FIG. 7. Integrated yields at midrapidity for pions, kaons, and antiprotons as a function of the charged particle density $\left(d N_{\mathrm{ch}} / d \eta\right)$, which is used as a measure of centrality. For comparison, $\mathrm{Au}+\mathrm{Au}$ data are shown as bands. Filled (open) points or bands depict data at $\sqrt{s_{N N}}=200 \mathrm{GeV}(62.4 \mathrm{GeV})$. Error bars represent statistical and systematic uncertainties added in quadrature. 


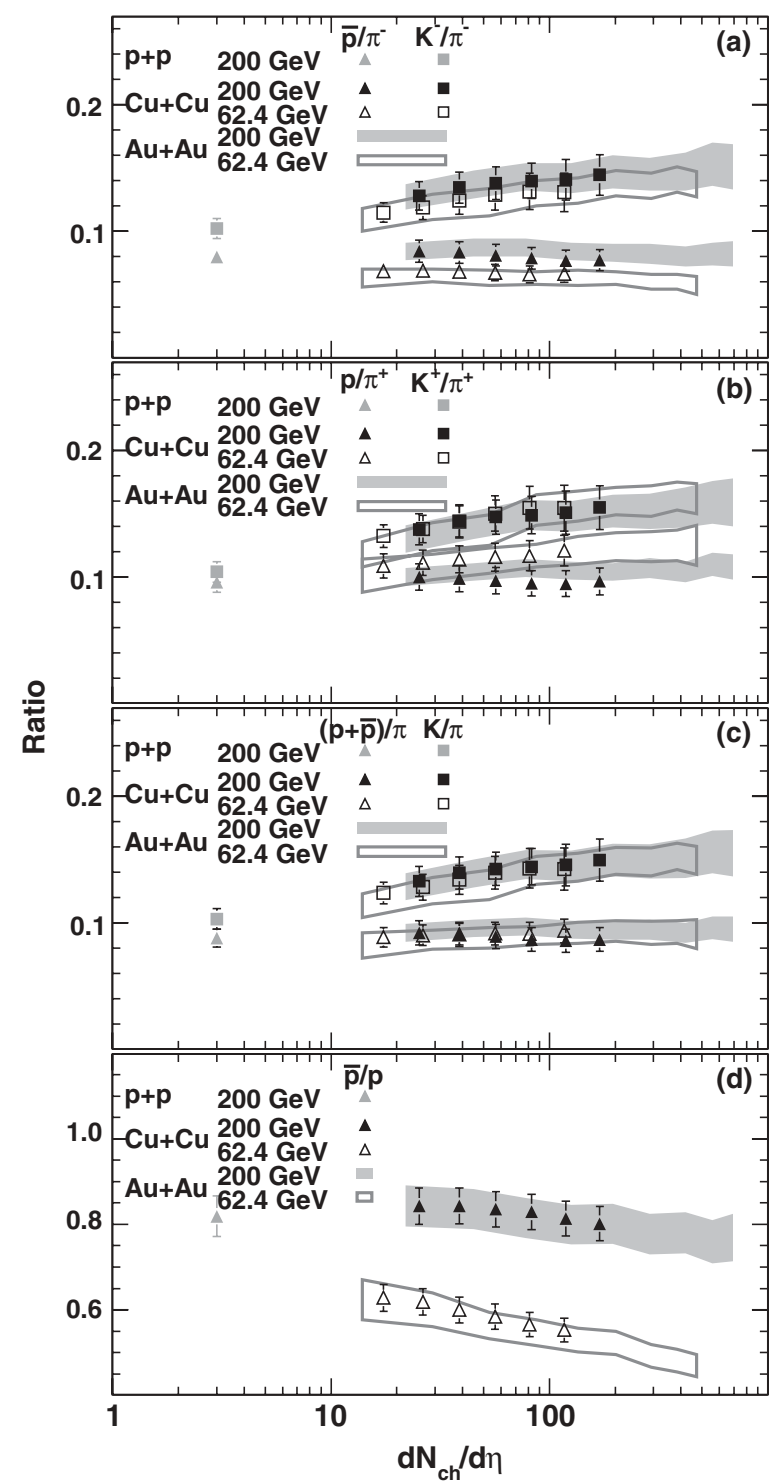

FIG. 8. Integrated particle yield ratios at $\sqrt{s_{N N}}=200 \mathrm{GeV}$ (closed symbols) and $62.4 \mathrm{GeV}$ (open) for $\mathrm{Cu}+\mathrm{Cu}$ (black) and $\mathrm{Au}+\mathrm{Au}$ collisions (gray bands) versus $d N_{\mathrm{ch}} / d \eta$ at midrapidity. Error bars represent statistical and systematic uncertainties added in quadrature.

centrality is evident, as shown earlier by the $\mathrm{K} / \pi$ ratios [Figs. 8(a) and 8(b)]. A comparison of these enhancement factors between $\mathrm{Cu}+\mathrm{Cu}$ and $\mathrm{Au}+\mathrm{Au}$ data is also shown. The enhancement factors for kaons do not show universal scaling features with respect to $N_{\text {part }}$ and are indeed found to be higher in $\mathrm{Cu}+\mathrm{Cu}$ collisions compared to the $\mathrm{Au}+\mathrm{Au}$ system. However, these features do not appear to be unique to kaons. A similar trend is observed in Fig. 9 in the pion enhancement factors for the two systems. This suggests that the additional enhancement, seen in the charged kaon yields, is not related to strangeness production, but other physics mechanisms; for example, additional entropy production. It should be noted that, while comparing more spherical central $\mathrm{Cu}+\mathrm{Cu}$ collisions with semiperipheral $\mathrm{Au}+\mathrm{Au}$ collisions, the initial conditions may not be reflected by $N_{\text {part }}$ alone.
In contrast to pions and kaons, protons show minimal evolution with centrality, and no difference between $\mathrm{Cu}+\mathrm{Cu}$ and $\mathrm{Au}+\mathrm{Au}$ systems is observed. Figure 8(d) illustrates the difference in antiproton and proton production across energies. As observed in other collision systems, the ratio is found to increase and becomes closer to unity for higher energy collisions [19]. The antiproton to proton ratio gives information on the amount of baryon transport. In line with the earlier STAR results, our measurements indicate that, while a finite excess of baryons over antibaryons is still present at RHIC energies, $p-\bar{p}$ pair production becomes an important factor. Little or no change due to an increase in the system size (centrality) is apparent in the $\mathrm{Cu}+\mathrm{Cu}$ data at $200 \mathrm{GeV}$, while 62.4-GeV data show a decreasing trend with increasing centrality for this ratio for both $\mathrm{Cu}+\mathrm{Cu}$ and $\mathrm{Au}+\mathrm{Au}$ data.

\section{FREEZE-OUT PROPERTIES}

The particle yields and their ratios provide further information on the thermal properties of the system at kinetic and chemical freeze-out.

\section{A. Kinetic properties}

The completion of all elastic scattering marks the final stage of collision evolution and could be interpreted as a kinetic freeze-out, where the particle momentum spectra are fixed. To quantify this stage, fits are made simultaneously to the spectra of all particle species, but independently for each centrality class (see, for example, Fig. 5). The fits used here are based on the previously discussed blast-wave model [4], which assumes a radially boosted thermal source. These hydrodynamically motivated fits describe the mass dependence of particle spectral shapes in terms of the radial flow velocity $(\beta)$, the kinetic freeze-out temperature $\left(T_{\text {kin }}\right)$, and the flow velocity profile exponent $(n)$ at the final freeze-out. The extracted value for $n$ is not used to derive any physics interpretation. The effects from resonance contributions to the pion spectral shape are reduced by excluding the low- $p_{T}$ data points (below $0.5 \mathrm{GeV} / c$ ). To enable a comparison with earlier results on $p p$ and $\mathrm{Au}+\mathrm{Au}$ collisions [5,7], the same model and the same procedures for the fits are adopted, thereby avoiding any possible systematic bias.

The blast-wave-fit results for the temperature of freeze-out are shown in Fig. 10. $T_{\text {kin }}$ and $\beta$ show similar dependencies as a function of $d N_{\mathrm{ch}} / d \eta$ in both $\mathrm{Cu}+\mathrm{Cu}$ and $\mathrm{Au}+\mathrm{Au}$ collisions, evolving smoothly from the lowest to the highest multiplicity, from $p p$ to central $\mathrm{Au}+\mathrm{Au}$. $T_{\text {kin }}$ decreases smoothly with centrality, implying that freeze-out occurs at a lower temperature in more central collisions. The similarity of kinetic freeze-out parameters in the events with similar multiplicity from different colliding species is confirmed by the data alone. As noted earlier, the particle mean $p_{T}$ increases with increasing $d N_{\mathrm{ch}} / d \eta$, which is consistent with an increase of radial flow with centrality. We note, however, that other physics mechanisms - for example, hard and semi-hard scatterings - can contribute to higher mean- $p_{T}$ values observed for kaon and proton spectra [20]. Direct spectral-shape 


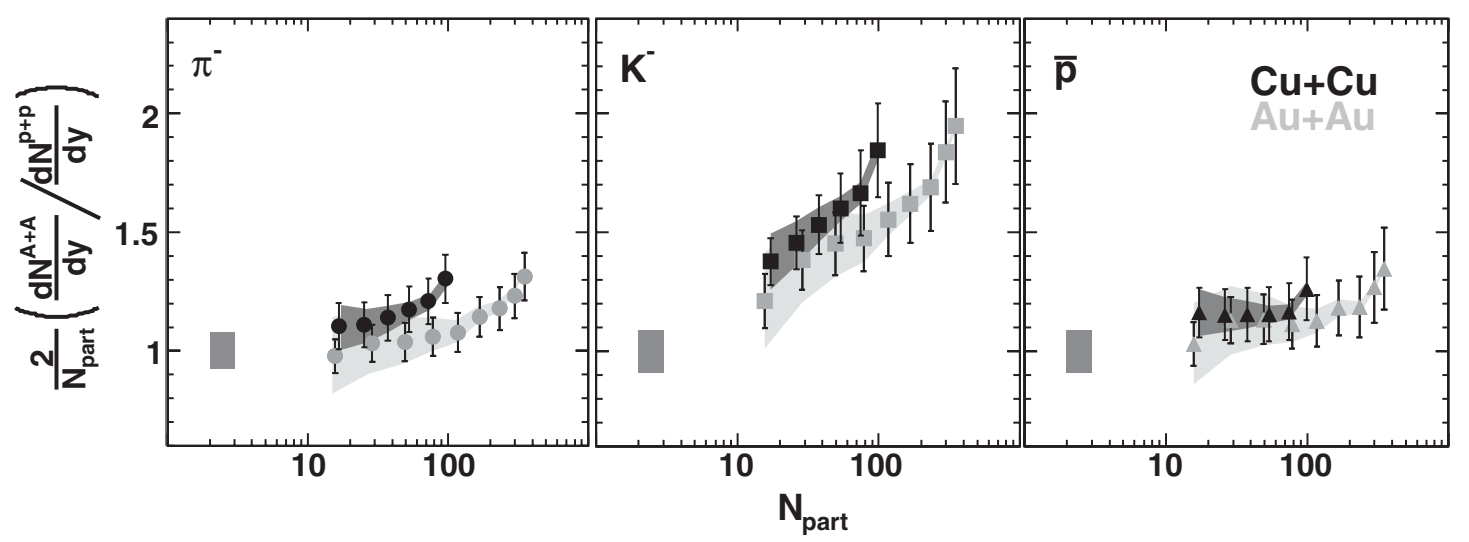

FIG. 9. Enhancement factors for negatively charged pions (left), kaons (center), and antiprotons (right) as function of $N_{\text {part }}$ in $\sqrt{s_{N N}}=200$ $\mathrm{Cu}+\mathrm{Cu}$ and $\mathrm{Au}+\mathrm{Au}$ collisions. Error bars represent statistical and systematic uncertainties on the A $+\mathrm{A}$ measurements added in quadrature. The shaded bands depict model uncertainties on number of participants calculation. The bands on the left show uncertainties from the $p p$ measurements that are correlated for all data points.

comparisons of $\mathrm{Cu}+\mathrm{Cu}$ and $\mathrm{Au}+\mathrm{Au}$ events from similar multiplicity bins, shown in Fig. 11, show the same $p_{T}$ dependencies between pion spectra from the two systems. The same is seen to hold for the respective kaon and proton spectra. The middle panel of Fig. 10 shows, in addition, the chemical freeze-out temperatures for different colliding systems at different energies. Both the chemical freeze-out and the kinetic freeze-out temperature show similar scaling features, reflecting the common trends in mean $p_{T}$ and the ratios of $p / \pi$ and $\mathrm{K} / \pi$, discussed earlier. Similarly, on the right panel of Fig. 10 we observe a common $d N_{\mathrm{ch}} / d \eta$ dependence for the average radial flow velocity at kinetic freeze-out.

A more important observation is that the obtained kinetic freeze-out parameters for pions, kaons, and (anti)protons follow the same trends with $d N_{\mathrm{ch}} / d \eta$, independent of collision energy, even though the production cross sections of the underlying spectra are different. This observation reflects a common evolution of charged-particle yields and mean $p_{T}$ for pions, kaons, and (anti)protons in collisions of different incident beams and centralities. A possible explanation can be furnished if kinetic freeze-out properties are determined by the initial state. For example, if one relates the total number of charged particles produced with the initial gluon density of the colliding system as in [21], then the freeze-out properties could be determined by the initial energy density of the collision.

\section{B. Chemical properties}

Chemical freeze-out occurs at the stage of the collision when all inelastic interactions cease and the produced particle composition in terms of yields is fixed. Valuable information for this collision stage can be obtained directly from the experimental results by forming particle ratios and comparing them across different collision systems and energies.

The ratios of the different particle yields in $\mathrm{Cu}+\mathrm{Cu}$ collisions are further analyzed within the framework of the statistical model [3]. This model describes the chemical freeze-out of the colliding system by several fit parameters: the temperature at which freeze-out occurs $\left(T_{\mathrm{ch}}\right)$, the cost of producing matter in terms of baryon and strangeness chemical potentials $\left(\mu_{\mathrm{B}}, \mu_{\mathrm{S}}\right)$, and an additional ad-hoc parameter, known as the strangeness suppression factor, $\left(\gamma_{\mathrm{s}}\right)$, to reconcile the
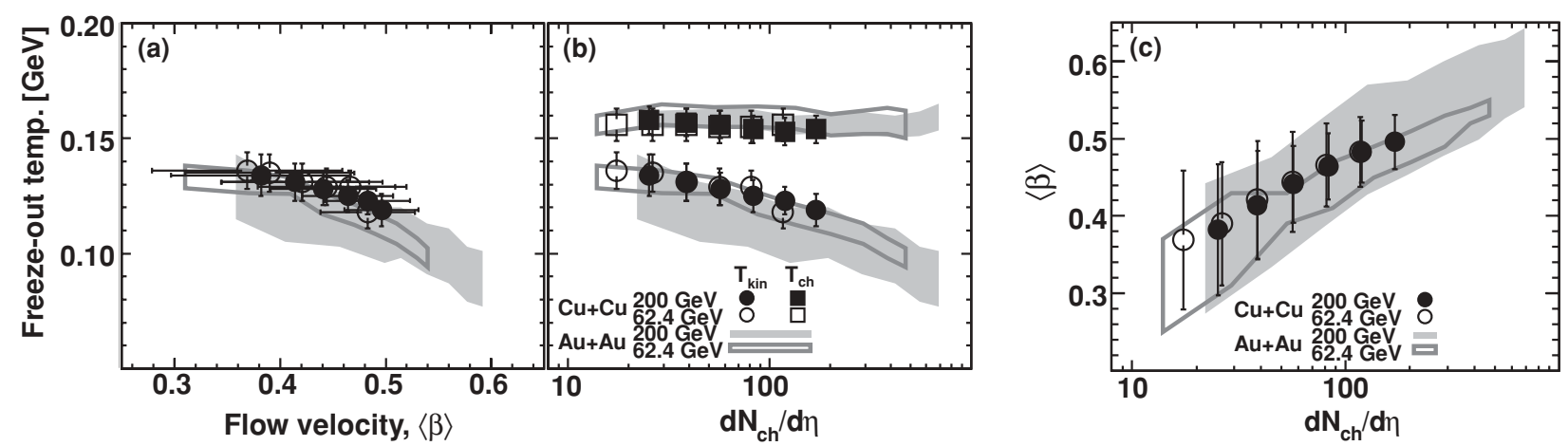

FIG. 10. Comparison of kinetic freeze-out properties obtained from fits to $\mathrm{Cu}+\mathrm{Cu}$ (symbols) and $\mathrm{Au}+\mathrm{Au}$ (bands) collision data at $\sqrt{s_{N N}}=200$ (closed symbols or bands) and $62.4 \mathrm{GeV}$ (open). The kinetic freeze-out temperature $T_{\text {kin }}$ is shown versus flow velocity $\beta$ and multiplicity in panels (a) and (b), respectively (more central collisions are to the right side of each plot). Panel (b) also shows the multiplicity dependence of the chemical freeze-out temperature $T_{\mathrm{ch}}$ (square symbols). Panel (c) shows the multiplicity dependence of the average radial flow velocity. 

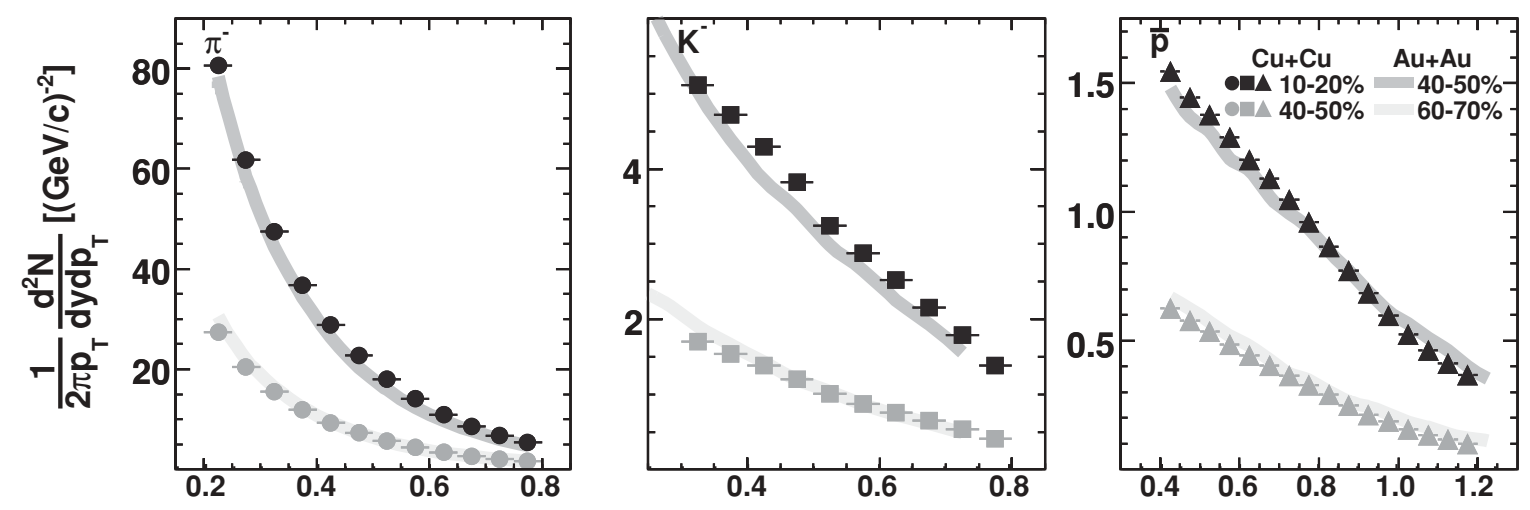

Transverse momentum, $p_{T}[\mathrm{GeV} / \mathrm{c}]$

FIG. 11. Comparison of the spectral shape between $\mathrm{Cu}+\mathrm{Cu}$ and $\mathrm{Au}+\mathrm{Au}$ data at $\sqrt{s_{N N}}=200 \mathrm{GeV}$. Centrality classes are chosen with a similar average charged hadron multiplicity at midrapidity. Pion (left), kaon (center), and antiproton (right) spectra are shown for $10 \%-20 \%$ central (40\%-50\%) $\mathrm{Cu}+\mathrm{Cu}$ (symbols) compared to 40\%-50\% midperipheral (60\%-70\%) $\mathrm{Au}+\mathrm{Au}$ (lines).

lower yield of strange hadrons in collisions involving smaller species (for example $p p$ and $\mathrm{d}+\mathrm{Au}$ ).

These statistical fits are performed on the relative particle abundances from $\pi^{ \pm}, \mathrm{K}^{ \pm}$, and $p(\bar{p})$ alone. Figure 12 shows an example of the resultant fit to the identified hadron ratios from central $\sqrt{s_{N N}}=200 \mathrm{GeV} \mathrm{Cu}+\mathrm{Cu}$ collisions. The lower panel of this figure illustrates the fit quality. We note that the successful description of the ratios by the model could not prove the attainment of chemical equilibrium, but suggests the statistical nature of particle production in these collisions [23]. The results obtained for the freeze-out parameters are shown in Figs. 10, 13, and 14.

Statistical model fits to a wider variety of hadron yields were also attempted using preliminary results for the $\Lambda, \Xi$, and $\phi$ particles and antiparticles from $200-\mathrm{GeV} \mathrm{Cu}+\mathrm{Cu}$ data from [14]. Including more particles into the model fits reduces the systematic uncertainty in the extracted parameters and resulted

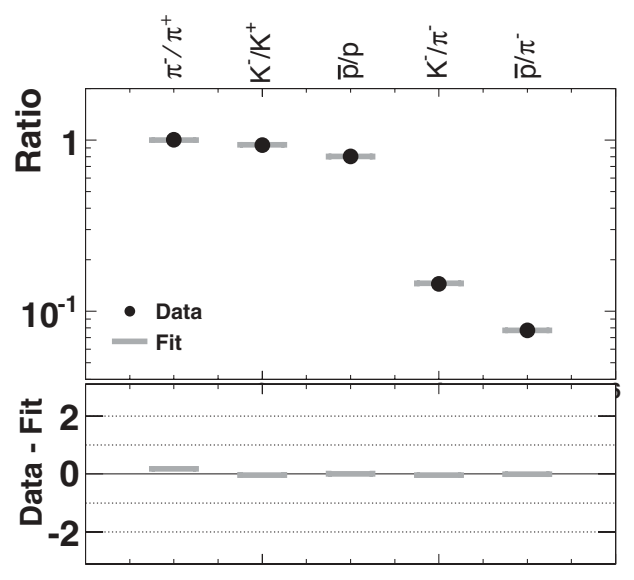

FIG. 12. The upper panel shows statistical-model-fit predictions (gray lines) for the measured particle ratios (circles) from central $200-\mathrm{GeV} \mathrm{Cu}+\mathrm{Cu}$ collisions. The lower panel illustrates the fit quality by showing the difference between the measured data and the model prediction in terms of the number of standard deviations $\left(N_{\sigma}\right)$ determined by systematic (data) uncertainty. in parameter values consistent with those obtained from fits using $\pi^{ \pm}, \mathrm{K}^{ \pm}$, and $p(\bar{p})$ alone; reported here. In general, the observed systematic trends in the freeze-out parameters as a function of the collision centrality are preserved [2,7].

Figure 14 (left panel) shows the evolution of the chemical freeze-out temperature versus baryon chemical potential in central heavy-ion collisions from the very low energy Heavy Ion Synchrotron (Schwerionensynchroton from German) (SIS) data through Alternating Gradient Synchrotron (AGS) and SPS to RHIC (STAR data points only). The overall evolution of $T_{\text {ch }}$ can be reproduced by the phenomenological model fit [22] applied here to all the data points shown (dashed line). As the collision energy increases, the temperature at freeze-out is found to increase up to SPS energies. This is followed by a plateau at RHIC energies at a value close to that of the hadronization temperature expected from lattice QCD calculations. At RHIC, for all systems and center-of-mass

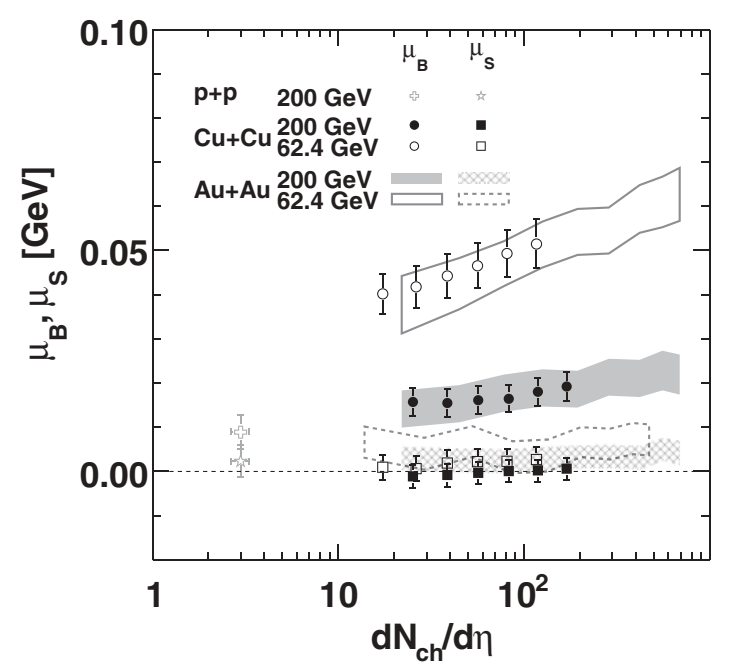

FIG. 13. Baryon and strangeness chemical potentials $\mu_{\mathrm{B}}$ and $\mu_{\mathrm{S}}$ as a function of $d N_{\mathrm{ch}} / d \eta$ for 200 and $62.4 \mathrm{GeV}$ in $\mathrm{Cu}+\mathrm{Cu}$ (symbols) and $\mathrm{Au}+\mathrm{Au}$ collisions (bands). 


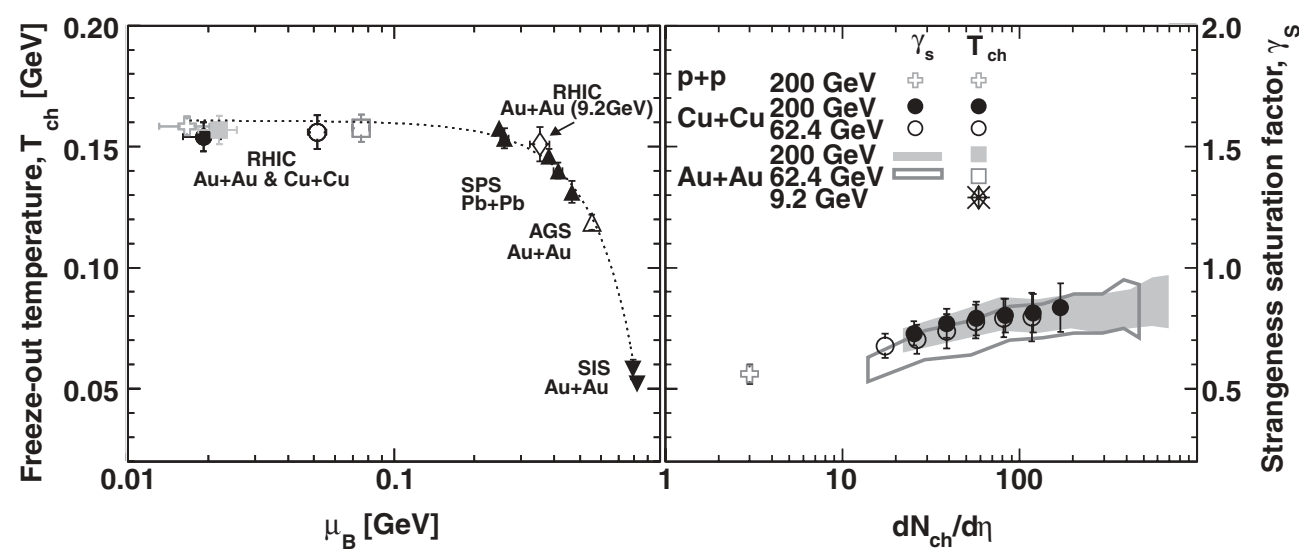

FIG. 14. Left: chemical freeze-out temperature $T_{\mathrm{ch}}$ as function of the baryon chemical potential $\mu_{\mathrm{B}}$ derived for central Au $+\mathrm{Au}(0 \%-5 \%$ for 200 and $62.4 \mathrm{GeV}$ [7] and $0 \%-10 \%$ for $9.2 \mathrm{GeV} \mathrm{[16])} \mathrm{and} \mathrm{Cu}+\mathrm{Cu}(0 \%-10 \%)$ collisions. For comparison, results for minimum-bias $p p$ collisions at $200 \mathrm{GeV}$ are also shown along with additional heavy-ion data points compiled for lower collision energies [22]. The dashed line represents a common fit to all available heavy-ion data described in the text. Right: strangeness suppression factor $\gamma_{\mathrm{S}}$ as a function of $d N_{\mathrm{ch}} / d \eta$ for 200 and $62.4 \mathrm{GeV}$ in $\mathrm{Cu}+\mathrm{Cu}$ (symbols) and $\mathrm{Au}+\mathrm{Au}$ collisions (bands).

energies, $T_{\text {ch }}$ appears to be universal, as shown in Fig. 10 (middle panel).

The value of the baryon chemical potential at a given centerof-mass energy is found to be slightly higher for the larger system, with $\mathrm{Au}+\mathrm{Au}$ and $\mathrm{Cu}+\mathrm{Cu}$ measurements showing common trends with charged hadron multiplicity (Fig. 13). We note that, presented in the same figure, values of strangeness chemical potential are close to zero with no obvious systematic trends for all energies and colliding systems studied at RHIC. Within a given system, $\mu_{\mathrm{B}}$ reflects the decrease in net-baryon density with increasing collision energy from $\sqrt{s_{N N}}=62.4$ to $200 \mathrm{GeV}$. This behavior can be observed directly from the particle ratios, where $\bar{p} / p$ increases as a function of energy (Fig. 8). For the most central $\mathrm{Cu}+\mathrm{Cu}$ events we measure $\bar{p} / p=0.80 \pm 0.04$ at $200 \mathrm{GeV}$ and $0.55 \pm 0.03$ at $62.4 \mathrm{GeV}$. The lack of centrality dependence in the baryon to meson ratios in $\mathrm{Cu}+\mathrm{Cu}$ and $\mathrm{Au}+\mathrm{Au}$ data points to similar freezeout temperatures for the studied systems. The constant values of $T_{\mathrm{ch}}$ at RHIC energies for collisions with different initial conditions, energy, and net-baryon density points to a common hadronization temperature of the systems.

Another parameter extracted from the fit, which is related to strangeness production, is the strangeness suppression factor $\gamma_{\mathrm{s}}$, shown versus $d N_{\mathrm{ch}} / d \eta$ in Fig. 14. The suppression of strange hadron yields is observed in smaller systems, such as $p p$ and peripheral collisions. Within statistical models this can be explained by a reduced production volume [24]. At low beam energies, where equilibration of $s$ quarks with respect to $u$ and $d$ is not expected, the suppression is also seen. We find that, within the systematic errors on the fit parameters, the strangeness suppression factor in $\mathrm{Cu}+\mathrm{Cu}$ is consistent with that for $\mathrm{Au}+\mathrm{Au}$ for the same number of charged particles, $d N_{\mathrm{ch}} / d \eta$. As only charged kaon yields were included in the fit, this observation is directly related to an absence of any additional enhancement in $\mathrm{K} / \pi$ at the same $d N_{\mathrm{ch}} / d \eta$ in the smaller $\mathrm{Cu}+\mathrm{Cu}$ system with respect to the larger $\mathrm{Au}+\mathrm{Au}$ system, as discussed previously.
The $\gamma_{\mathrm{s}}$ parameter shows a similar increase with centrality for both systems and energies. The value of $\gamma_{\mathrm{s}}$ approaching unity for the central $\mathrm{Au}+\mathrm{Au}$ collisions in the context of thermal model would imply that the produced strangeness is close to equilibrium.

\section{SUMMARY}

We have presented measurements of identified charged hadron spectra in $\mathrm{Cu}+\mathrm{Cu}$ collisions for two center-of-mass energies: 200 and $62.4 \mathrm{GeV}$. These new results of $\pi^{ \pm}, \mathrm{K}^{ \pm}$, and $p(\bar{p})$ have further enriched the variety of low- $p_{T}$ spectra at RHIC. The data have been studied within the statistical hadronization and blast-wave-model frameworks in order to characterize the properties of the final hadronic state of the colliding system as a function of system size, collision energy, and centrality.

These multidimensional systematic studies reveal remarkable similarities between the different colliding systems. No additional enhancement of kaon yields with respect to pions is observed for the smaller $\mathrm{Cu}+\mathrm{Cu}$ system compared to $\mathrm{Au}+\mathrm{Au}$. The obtained particle ratios, mean $p_{T}$ and the freezeout parameters, including the strangeness suppression factor $\gamma_{\mathrm{s}}$, are found to exhibit a smooth evolution with $d N_{\mathrm{ch}} / d \eta$, and similar properties at the same number of produced charged hadrons are observed for all collision systems and center-ofmass energies. A notable exception in our study is the absence of $d N_{\mathrm{ch}} / d \eta$ scaling for the $\bar{p} / p$ ratio (and the closely related baryon chemical potential $\mu_{\mathrm{B}}$ ). The bulk properties studied have a strong correspondence with the total particle yield. Within thermal models this reflects a relationship between the energy per particle at freeze-out and the entropy derived from particle yields. The baryon chemical potential could, in addition, be influenced by the initial valence quark distribution and by baryon transport during expansion, leading to a more complicated dependence. The scaling features of freeze-out properties are not presented at the same $N_{\text {part }}$ for lighter and heavier ions as scaling is badly broken when data measured at 
different energies are compared. This suggests that $N_{\text {part }}$ does not fully reflect the initial state of the system.

\section{ACKNOWLEDGMENTS}

We thank the RHIC Operations Group and RCF at BNL, the NERSC Center at LBNL, and the Open Science Grid consortium for providing resources and support. This work was supported in part by the Offices of NP and HEP within the US DOE Office of Science, the US NSF, the Sloan Foundation, the DFG cluster of excellence "Origin and Structure of the Universe" of Germany, CNRS/IN2P3, STFC and EPSRC of the United Kingdom, FAPESP CNPq of Brazil, Ministry of Ed. and Sci. of the Russian Federation, NNSFC, CAS, MoST, and MoE of China, GA and MSMT of the Czech Republic, FOM and NWO of the Netherlands, DAE, DST, and CSIR of India, Polish Ministry of Sci. and Higher Ed., Korea Research Foundation, Ministry of Sci., Ed. and Sports of the Rep. of Croatia, Russian Ministry of Sci. and Tech. and RosAtom of Russia.
[1] F. Karsch, J. Phys. Conf. Ser. 46, 122 (2006).

[2] J. Adams et al., Nucl. Phys. A 757, 102 (2005).

[3] P. Braun-Munzinger, I. Heppe, and J. Stachel, Phys. Lett. B 465, 15 (1999).

[4] E. Schnedermann, J. Sollfrank, and U. Heinz, Phys. Rev. C 48, 2462 (1993).

[5] J. Adams et al., Phys. Rev. Lett. 92, 112301(2004).

[6] L. Molnar et al., Acta Phys. Hung. A 25, 293 (2006).

[7] B. Abelev et al., Phys. Rev. C 79, 034909 (2009).

[8] F. Karsch, Nucl. Phys. A 698, 199 (2002).

[9] O. Barannikova et al., arXiv:nucl-ex/0403014.

[10] J. Adams et al., Phys. Rev. Lett. 92, 171801 (2004).

[11] C. Adler et al., Nucl. Instrum. Meth. A 470, 488 (2001).

[12] M. Anderson et al., Nucl. Instrum. Meth. A 499, 659 (2003).

[13] W. M. Yao et al. (Particle Data Group), J. Phys. G 33, 1 (2006).

[14] A. Timmins et al., Nucl. Phys. A 830, 829 (2009).
[15] J. Takahashi and R. Derradi de Souza, arXiv:0809.0823v1 [nucl-ex].

[16] B. Abelev et al., Phys. Rev. C 81, 024911 (2010).

[17] C. Hohne, Nucl. Phys. A 715, 474 (2003).

[18] C. Alt et al., Phys. Rev. Lett. 94, 052301 (2005).

[19] B. B. Back et al., Phys. Rev. C 71, 021901 (2005).

[20] T. Trainor, Int. J. Mod. Phys. E 17, 1499 (2008).

[21] L. McLerran, Acta Phys. Polon. B 34, 3029 (2003); D. Kharzeev and E. Levin, Phys. Lett. B 523, 79 (2001); D. Kharzeev, E. Levin, and L. McLerran, ibid. 561, 93 (2003).

[22] J. Cleymans, H. Oeschler, K. Redlich, and S. Wheaton, Phys. Rev. C 73, 034905 (2006); F. Becattini, J. Cleymans, A. Keranen, E. Suhonen, and K. Redlich, ibid. 64, 024901 (2001).

[23] F. Becattini and G. Pettini, Phys. Rev. C 67, 015205 (2003).

[24] J. Cleymans, K. Redlich, and E. Suhonen, Z. Phys. C 51, 137 (1991). 